\section{Toxic assets}

\section{By C. Simone Fishburn, Senior Editor}

A new tool for in vivo detection of liver toxicity could represent a significant improvement over routine methods that only work in vitro or on tissue slices. ${ }^{1}$ The litmus test for the Stanford University School of Medicine inventors will be to show that the nanoparticle-based method can detect toxicity in compounds that previously eluded standard analysis and later failed in the clinic.

Current preclinical toxicity testing methods are clearly suboptimal, as more than $20 \%$ of compounds end up failing clinical trials because of toxicity issues. ${ }^{2}$

Standard toxicology analysis involves early stage screening using in vitro microsomes, hepatocytes and liver slices followed by INDenabling histopathology and plasma biomarker studies on animals treated for two weeks or longer.

IND submissions also require additional assessments such as genotoxicity and immunotoxicity.

Multiple tools have been tried in discovery research, but few have been incorporated into routine preclinical toxicology assessment.

Michael Taylor, founder and principal of NonClinical Safety Assessment, told SciBX that the field often is resistant to disruptive innovation. Companies prefer to focus resources on techniques currently accepted by the FDA, and toxicologists want to see a validated improvement over existing methods before switching to a new one.

"Toxicology is in general a very risk-averse field, so it's a tough nut to crack with this space-even for new tools that are exciting," he said.

Attitudes aside, it is very difficult to monitor short-lived metabolites in vivo; thus, toxicity analysis relies on detecting biomarkers in isolated plasma or tissue samples.

Jianghong Rao, an associate professor of radiology and chemistry at the Stanford University School of Medicine, encountered that very problem about three years ago when he was studying markers related to inflammation.

$\mathrm{He}$-like other researchers-was trying to find short-lived markers of toxicity. "The big challenge was that you could only apply reagents locally or in cell culture, but you couldn't apply them systemically to see inflammatory sites in vivo," he told SciBX.

As luck would have it, others in his lab had developed a technology based on semiconducting polymer nanoparticles (SPNs) for deeptissue imaging of tumors. Those particles could detect signals with high sensitivity and had good in vivo stability and distribution properties.

That led the team, which included researchers from the University of Toronto, to adapt the technology to detect-in live animals-shortlived biomarkers that are inaccessible to standard techniques.

\section{Interactive sensors}

Because drug-induced toxicity occurs primarily in the liver, Rao's team focused on reactive metabolites formed as transient by-products of hepatic clearance enzymatic reactions.

Reactive oxygen species (ROS) are generated by oxidative enzymes such as cytochrome P450 (p450) or by reactions of drug metabolites with oxygen. Reactive nitrogen species (RNS) are the result of drug metabolite-induced disruption of the electron transport chain, which leads to mitochondrial toxicity.

ROS and RNS thus represent two potential biomarkers of toxicity produced by different mechanisms that have different biological effects.

To enable real-time simultaneous monitoring of hepatic ROS and RNS in live rats, the team designed SPNs containing two matrix polymers, two sensors and a liver-targeting, galactose-conjugated polyethylene glycol (PEG) tail.

The ROS sensor in the matrix is a chemiluminescent substrate that breaks down in the presence of the ROS hydrogen peroxide to produce a reaction intermediary that excites nearby dye molecules to emit light.

Via chemiluminescence resonance energy transfer (CRET), the light emitted serves as an excitation source for the fluorescent RNS sensor.

When no RNS is present, the fluorescent sensor embedded in the matrix responds to the excitation source and emits light at wavelengths of $680 \mathrm{~nm}$ and $820 \mathrm{~nm}$ via fluorescence resonance energy transfer (FRET). However, when the RNS peroxynitrite is present, the sensor dye decomposes and the FRET is lost-along with the $820 \mathrm{~nm}$ emission. The $680 \mathrm{~nm}$ emission increases as a consequence.

Thus, ROS measurement involves direct quantitation of the luminescence signal, and RNS measurement is based on alterations in the ratio of the $680 \mathrm{~nm}$ and $820 \mathrm{~nm}$ emissions.

To test the nanoparticles, the researchers gave mice subtoxic to toxic doses of acetaminophen. Overdoses of that drug cause necrotic cell death through overproduction of ROS and RNS.

The team captured sequential fluorescence and luminescence images for 80 minutes after dosing. ROS and RNS were detected 18 minutes and 53 minutes after a toxic $300 \mathrm{mg} / \mathrm{kg}$ acetaminophen dose. Only baseline signals were seen at all the lower doses.

That threshold type of toxicity -in which toxicity only occurs above a specific dose level-is characteristic of acetaminophen and differs from dose-dependent toxicity in which increasing levels of toxic products are formed as the dose increases.

The nanoparticle method produced evidence of toxicity earlier than immunohistochemical and histological analysis of liver tissue. Those methods showed no changes at 45 minutes and only much later-at 180 minutes-showed signs of necrosis.

Glutathione, a scavenger of reactive metabolites, reversed the signals produced by the acetaminophen-generated ROS and RNS. That finding suggested that the technique also could be used to assess agents that help reverse or recover from toxicity.

Finally, the researchers tested whether the SPNs could detect ROS and RNS produced by isoniazid, a widely used tuberculosis drug that causes significant hepatotoxicity by poorly understood mechanisms.

Because the kinetics and sequence of ROS and RNS generation depend 
on which metabolic pathway is involved, the group expected that a timecourse study would provide clues to the mechanism of toxicity.

As with acetaminophen, the SPNs detected both hydrogen peroxide and peroxynitrite from isoniazid. Unlike acetaminophen, the two toxicity readouts produced very different time profiles. The results suggested that isoniazid toxicity occurs mainly through a nitrosylation pathway, whereas acetaminophen metabolism involves oxidation and nitrosylation.

The kinetics also showed that isoniazid toxicity is dose dependent-in contrast to the threshold type of toxicity seen for acetaminophen.

The authors concluded that the SPNs can be used to help understand mechanisms of toxicity and that the technology can be used for in vivo monitoring and early detection of potentially toxic preclinical candidate compounds.

Results were published in Nature Biotechnology.

\section{Translational toxicology}

Rao told SciBX that the team is testing the technology on a range of molecules in animals. He also plans to develop it for clinical use, in particular for monitoring liver damage in patients waiting to receive transplants.

According to Jonathan Sorger, the technology is a big step toward solving the problem of finding biomarkers for liver imaging. "Everyone is trying to come up with new biomarkers: early, predictive and noninvasive are the key properties to have," he said.

Sorger said that the ability to take serial measurements in intact animals is a key advantage of the new method.

"I'm not sure if this will be the way to do it in the end, but it's a better way than what we're doing now," he said. He added that in drug screening, the SPN technology would most likely be used after standard in vitro tests with microsomes and hepatocytes.

Sorger is director of medical research at Intuitive Surgical Inc., a medical device company with imaging technologies for noninvasive surgery.

Alexandra de Lille agreed that the noninvasive aspect of the new

"It's very much in the pioneer stage to use multiplereporter systems in the same animal-you get two outcomes from one mouse. Technically we could probably get five readings out of one mouse with a system like this. In the past it has been one reading per mouse."

- Alexandra de Lille, technique is one of its biggest advantages over current methods as it can detect toxicity biomarkers and perform kinetic assessments without "slicing and dicing of tissues."

She said that in addition to providing mechanistic clues underlying a toxic readoutsuch as whether nitrosylation or oxidation pathways are dominant-the information from kinetic monitoring can translate to dosing decisions.

de Lille is director of technical applications for in vivo imaging at

Caliper Life Sciences, a unit of PerkinElmer Inc.

She also liked that the technology can monitor multiple toxic metabolites in parallel.

"It's very much in the pioneer stage to use multiple-reporter systems in the same animal-you get two outcomes from one mouse. Technically we could probably get five readings out of one mouse with a system like this. In the past it has been one reading per mouse," she told SciBX.
According to Sorger, the signal intensity used in the study works well in mice but would not be sufficient for use in humans.

"This makes a lot of sense in the drug screening world, but in humans it will be hard to get the necessary tissue penetration," he said. "In humans, photons [in this wavelength range] don't penetrate tissue far enough. You can go to $1 \mathrm{~cm}$ depth, but the liver is deeper than that. You would need to use a different emitter or a radioisotope."

de Ville agreed that use in humans is further in the future.

Taylor said that the method still has challenges to meet before it is used in animals because a more sophisticated readout does not necessarily translate to better predictive power.

He said that the team compared its method with standard histopathology but did not compare it with plasma biomarkers used in routine toxicology assessments.

The standard to beat, according to Taylor, is biomarkers of hepatotoxicity such as transaminase and superoxide dismutase (SOD).

He said that one way to show superiority would be to draw plasma and look at transaminase and SOD in addition to histopathology to see whether the Stanford group's method is more sensitive.

"Sometimes there's a disconnect between biomarkers such as transaminase and SOD and the visual histopathological tissue markers, so if they could show the technique can be a better predictor of tissue damage, that would be an advantage," said Taylor.

Another meaningful benefit, he said, would be getting reliable information faster than current methods.

"A two-week full rat toxicology study can approach $\$ 100,000$. So if you can get a signal sooner than that, there would be significant savings," he said.

Those savings involve not only time-related costs of the study but also the amount of material that needs to be synthesized, which is one of the most costly and rate-limiting aspects of preclinical development, he said. "This is one of the first big financial jumps for a small company," he added.

Taylor said that the real test will be to take compounds that were dropped in the clinic because of toxicity in humans and see if the new method can produce a preclinical toxicity signal where the current methods failed.

"To be a game changer, it needs to increase the chances of detecting toxicity better than other methods," he said.

Stanford University has filed a patent on the findings. The technology is available for licensing.

Fishburn, C.S. SciBX 7(14); doi:10.1038/scibx.2014.392

Published online April 10, 2014

\section{REFERENCES}

1. Shuhendler, A.J. et al. Nat. Biotechnol.; published online March 23, 2014; doi:10.1038/nbt.2838

Contact: Jianghong Rao, Stanford University School of Medicine, Stanford, Calif.

e-mail: jrao@stanford.edu

2. Kola, I. \& Landis, J. Nat. Rev. Drug Discov. 3, 711-715 (2004)

COMPANIES AND INSTITUTIONS

Intuitive Surgical Inc. (NASDAQ:ISRG) Sunnyvale, Calif.

NonClinical Safety Assessment, Mountain View, Calif.

PerkinElmer Inc. (NYSE:PKI), Waltham, Mass.

Stanford University, Stanford, Calif.

Stanford University School of Medicine, Stanford, Calif.

University of Toronto, Toronto, Ontario, Canada 